\title{
Muography, Outreaching, and Transdisciplinarity: Toward the Golden Age of Muography
}

\author{
Marko Holma, 1,2,3,4 Pasi Kuusiniemi, 1,2,3 and Jari Joutsenvaara 1,2,4 \\ ${ }^{1}$ Muon Solutions Oy, Pyhäjärvi, Finland \\ ${ }^{2}$ Kerttu Saalasti Institute, University of Oulu, Finland \\ ${ }^{3}$ International Virtual Muography Institute, Tokyo, Japan \\ ${ }^{4}$ Arctic Planetary Science Institute, Finland \\ Corresponding author: Marko Holma \\ Email:marko.holma@muon-solutions.com
}

\begin{abstract}
We demonstrate that cosmic-ray muography is a fundamentally multidisciplinary research field requiring an outreaching and transdisciplinary approach to support and speed up its current positive growth stage. The transit from expert-driven multidisciplinary research to interdisciplinary and transdisciplinary research requires publishing and promoting muography on multiple fronts and languages. Still, as the rewards for the muography community are likely great indeed, we call for collaborative actions and a change in the research strategy paradigm. Due to this end, we suggest a list of task points for the presentday muography community to get muography better acknowledged and as appealing as possible for the newcomers interested in developing muography or applying it in their respective applications.
\end{abstract}

Keywords: muons, muography, applied geophysics, science outreaching, multidisciplinarity, interdisciplinary, transdisciplinarity, research strategies

DOI: $10.31526 / J A I S .2022 .258$

\section{INTRODUCTION}

Muography is an umbrella term for a group of passive imaging techniques for material density. It relies on the penetration power of cosmic-ray-induced atmospheric muons, the technical capabilities of the muon detectors, the modern computing power, the effectiveness of image reconstruction algorithms, and the interpretation power of the multidisciplinary scientific community of muographers. Although it cannot be disputed that muography needs improvements on all these fronts before it can be considered equal among the scientific disciplines, it is perhaps the latter that needs to be progressed most to make that happen. Indeed, considering the wide range of muography applications and the need for making muography more suitable and available for the larger scientific and end-user communities, muography is, for its own benefit, currently a far too narrow research field.

This work focuses on the multidisciplinary attributes of muography and why more interdisciplinary discourse within the muography developers is needed, not forgetting the equally important tasks of outreach and transdisciplinarity actions. The broad conception of transdisciplinarity describes it as a form of research driven by the need to solve complex real-world problems and relates it to three types of knowledge: systems knowledge, target knowledge, and transformation knowledge [1]. In the light of the present work, the systems knowledge would correspond to the sum of all we currently know of muography and its application areas (i.e., knowledge about the current system or problem situation). This type of knowledge is mainly analytical and descriptive. The target knowledge would correspond to knowledge about the desired future (i.e., where we like to end up with). Lastly, the transformation knowledge is about how to make the shift from the current to the desired situation (i.e., what are the concrete strategies and steps to take to reach the target). As a whole, a transdisciplinary research strategy is a holistic approach for constructing project goals, formulating research questions, and planning actions. We have adopted the concept of transdisciplinary research herein to obtain a list of recommended activity steps for the present-day muography community to obtain more impactful results quicker by working together.

As the scope of the present paper is intimately associated with the recent developments in the publishing front, we have prepared a companion paper titled "Trends in publishing muography related research results. The situation at the end of 2020" [2], which further enlightens the herein discussed themes.

\section{THE BASICS OF COSMIC-RAY MUOGRAPHY}

Atmospheric muons are created in chain reactions triggered when cosmic rays collide with nuclei in the upper atmosphere. As a muon has a heavy mass and the atmospheric muons have relativistic speeds, muon particles (informally "muon rays") can 
penetrate several magnitudes deeper into material than the somewhat analogous X-rays. The latter are penetrable mere few meters only. Since muons are naturally created in the atmosphere, there is no need for particle accelerators or radiation sources (cf. X-ray generation). Yet, another difference between X-rays and muon rays is that the natural flux of the latter is typically remarkably lower than that of artificial X-rays. This fact renders muography a slow imaging method compared to optical and X-ray imaging techniques based on photons. In many other ways, muography can be compared to such imaging techniques as X-ray radiography and X-ray tomography.

Muographic imaging techniques can be shared into absorption- and scattering-based varieties. The common factors include passiveness, noninvasiveness, the harmlessness of the form of radiation exploited and the capability to detect a muon that has passed through the object of interest. Most detector systems can also record the direction of an incoming muon (i.e., resolve its straight flight track) with great accuracy. Still, in some cases, this feature is not required, or the detector geometry does not support it (e.g., cylindrical borehole detectors are often constrained with a lower angular resolution). Furthermore, two-dimensional muography data can also be used for reconstructing three-dimensional density models. These imaging modes are, respectively, termed muon radiography and muon tomography. Each of the two can also be performed as time-lapse (or time-sequential) imaging.

The muon detector geometries, shapes, and sizes are by necessity varied to carry out muography in absorption and scattering modes and in different physical environments (e.g., surface, underground, borehole, underwater, and airborne). There are also a multitude of variations in muon detection itself as this can be done, for example, with plastic scintillators [3], nuclear emulsions [4], drift chambers [5], micro-mesh gaseous structures [6], resistive plate chambers [7], and multiwire proportional chambers [8].

Thus, there is a relatively broad spectrum of different forms of muography and many ways to detect muons. Due to practical reasons, this has led to the differentiation and specialization of the various detector designer groups, research teams, and nascent private companies operating in the field of muography. Moreover, the muographers are widely scattered across continents, countries, and time zones. These natural boundaries have historically guided the development of muography and kept the working groups more or less isolated.

\section{WHY MUOGRAPHY NEEDS OUTREACHING AND TRANSDISCIPLINARITY}

The development history of muography started already in the 1950s [9], but it can easily be claimed that the current stage of rapid development was not commenced until the mid-2000s. The earlier stages of muography, years before the term "muography" (literally, "drawing with muons") was even coined by H. K. M. Tanaka [10], have been documented well elsewhere [11].

Based on the sheer number and content of muography-related publications in the last decade [12, 13], it is hard to argue against the conclusion that muography is currently experiencing a progressive growth stage that overpasses the speed of any of its earlier growth stages. Likewise, it can be reasoned that muography is not anymore the playing field for particle physicists and detector designers alone. This is easy to demonstrate by examining quantitatively the number of authors who published their first works on muography in recent years and assessing the scope of the titles of the publications. Indeed, muography is now clearly experiencing rapid growth and a stage of lateral expansion across the different fields of science and engineering.

The field of muography may appear challenging for newcomers due to (i) the wide range of detector designs, (ii) the number of modes of muography, (iii) unfamiliar vocabulary, (iv) unestablished practices relating to terms, (v) inconsistencies between authors how the present-day muographers use concepts and words, (vi) variations in the environments and applications muography can be used, and lastly, (vii) the very specifics of the astroparticle physics itself, such as the concepts and processes related to the hadronic air shower cascades, particle propagation, energy spectrums, muon angular distributions, stopping power, and simulations. We propose that this inherited challenge of muography can be overcome by transdisciplinary actions that will eventually aid in correcting the current disparity between fundamental physics papers and those of application-oriented articles. Only with a more significant number of review papers, case studies, and end-user-directed publications can the present muography community hope to get their research acknowledged and under greater acceptance. This also requires establishing more extensive and varied research consortia, preferably crossing borders and spanning more than one continent. The private sector needs engaging too.

However, even the aforementioned activities are not yet enough. Additional activities needed include versatile science outreach and publishing not only in English but also in the many other domestic languages the researchers represent. Moreover, it is recommendable to get the third-party science popularizers engaged in publishing updates and overviews of the recent papers and breakthroughs in muography. This, of course, cannot be implemented by influencing unethically; instead, as a community, we must look for ways to publish easy-to-read and easy-to-understand papers on our research and thereby make muography more appealing and approachable (granted, this work may not be the prime example).

A quantitive review of the popular science news sites on the web such as Phys.org and The New York Times' Science page supports a view that muography is on the verge of stepping into scientific adulthood. Although this conclusion may be biased, it is easy to verify by examining the nature and number of popularized articles on muography on many of these websites. The muographers themselves can aid in sustaining this positive trend by, when appropriate, publishing review papers on the benefits of muography in terms of, for example, societal issues, industrial productivity, and public safety. Publications on how muography can aid in solving other sciences' challenges are also needed. In addition, it is preferable that muographers are active in publishing muography-related results and novel ideas also in more popularized form than the standard expert-to-expert science journals. For example, blog postings and easy-to-grasp summaries in science magazines would make muography more welcoming for those science news reporters who have not yet heard of it. 


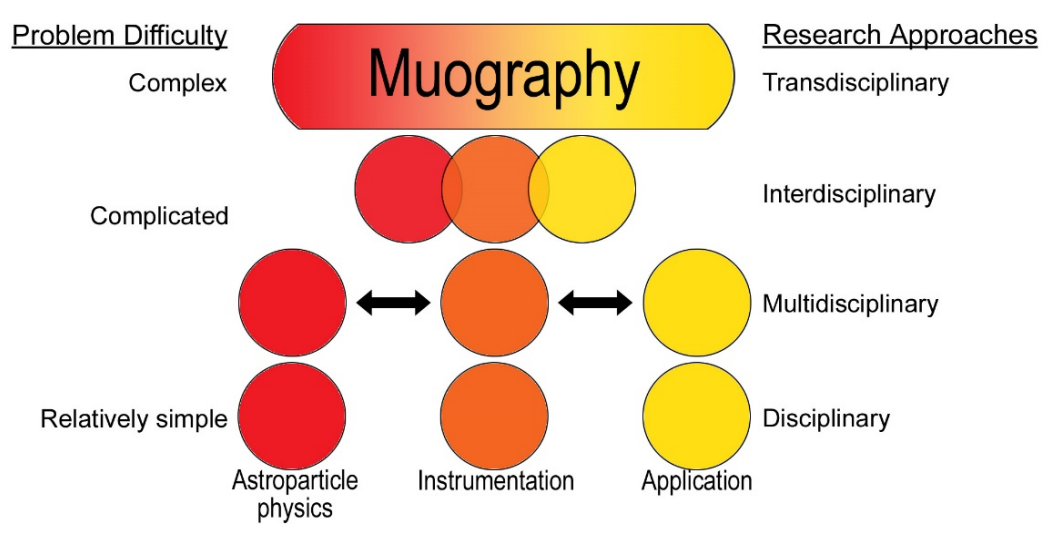

FIGURE 1: A conceptual diagram emphasizing the complexity of the real-world challenges met by the muography community and the appliers of muography. In our opinion, muography is fundamentally multidisciplinary, but benefits from interdisciplinary and transdisciplinary discourse and collaboration as these research approaches match best with the problem difficulty levels muography faces. The transdisciplinary science approach integrates or requires various disciplines, concepts, and methods to make research as productive as possible. Modified from [14].

\section{WHY MUOGRAPHY NEEDS OUTREACHING AND TRANSDISCIPLINARITY}

It can be reasoned that many emerging scientific disciplines arise when the conventional sciences cross paths in a manner not seen before. Events like these may lead to the rapid development of new methodologies, concepts, and technologies as the collaboration between previously separate research communities form a fruitful background to further developments. This is the case also with muography, which—at least occasionally_combines as varied fields of human activity as particle physics, detector designing, software coding, archaeology, architecture, border control, speleology, container imaging, nuclear waste imaging, volcanology, hydrology, geology, mineral exploration, mining, oceanography, and even arts, not mentioning a multitude of other geoscience and engineering applications. Moreover, the cross-pollination between different disciplines that do not naturally interact makes muography an exciting and rapidly developing field of research. It also drives emergent commercial activities.

Muography deviates from conventional particle physics by providing more visual results for the audience. It differs from conventional particle physics also by being an applying method rather than a fundamental and profoundly theoretical science. Many geophysical methods are used in geosciences, but only a few can be traced back to the quantum world of particle physics. Hence, the conventional curriculums of geophysicists and geologists, for example, are rarely preparing the graduating scientists for the requirements of muography. As muographers, it is our responsibility to further develop muography to overcome the barrier that still separates it from other geophysical subdisciplines. This can be done by active collaboration between the different research groups working in muography. However, as the applications of muography vary drastically from one to another, we also need effective communication with the researchers of other sciences. In addition, we need outreach projects that let us be seen, heard, and experienced in the media, science parks, and art installations by other scientists and the public alike. Some such actions have already been actualized.

Because muography applications are so drastically varied, muography is intrinsically multidisciplinary and requires an interdisciplinary or even a transdisciplinary approach to master. A transdisciplinary science approach integrates or requires various disciplines, concepts, and methods to make research as impactful as possible [1]. When the research problem is relatively simple (from the viewpoint of modern curriculums), or at least limitable, the conventional research fields are applied (e.g., astroparticle physics, instrumentation, and geoscience work and develop separately). However, when the challenges are more complicated, the different disciplines need interaction that can be described as multidisciplinary or interdisciplinary, depending on how much there is overlap. There are, nevertheless, also research fields that require even more interaction between different disciplines than the multidisciplinary approach can offer. We propose that muography is such an applied science (Figure 1).

Since atmospheric muons can be detected as rare particles in well over $1 \mathrm{~km}$ depth, it can—at least theoretically-be envisioned a future where muography is applied in relatively deep underground settings to the most diverse group of applications [12, 13]. Therefore, muography demands an interdisciplinary if not transdisciplinary research approach as data inversions and interpretations are not always straightforward. This is one more reason why muography needs more publicity and acknowledgment and end-users (i.e., appliers) who have been adequately trained in the principles of muography.

\section{CONCLUDING REMARKS AND TASK POINTS FOR INCREASING THE APPEAL OF MUOGRAPHY}

Muography is a multidisciplinary research and application field rather than a field of fundamental physics. This is best demonstrated that, depending on the case, a successful measurement campaign may need a combination of the following:

(i) detector know-how, 
(ii) understanding of particle physics,

(iii) simulation expertise,

(iv) software development skills,

(v) proficiency in practical field studies and related logistics,

(vi) expertise at the application-specific science and its traditions (e.g., correct use of science- and application-specific vocabulary).

In brief, muography cannot be carried out by a single person.

It must also be emphasized that as muography has a large number of proven and potential applications, its end-users are needed to get along. This, in turn, requires that muographers actively promote their science and methods for those of different backgrounds. This can be carried out with a combination of the following actions:

(i) Publications for different audiences and written in various languages are needed.

(ii) Many types of publications are needed, including review papers, case studies, end-user-directed publications, marketing brochures, white papers and even blogs.

(iii) Civil sector websites dedicated to science outreaching and popularization need to get interested and familiarized with the principles of muography.

(iv) Training courses on the principles and possibilities of muography are needed.

We thereby argue that the muography community must increase its science outreach activities. With these actions, muography will maintain its current positive development trend and become even more appealing, if not mainstream.

In our opinion, muography benefits the most if the muography community does its best to increase collaboration between the muographers and its present and conceived stakeholders. There have also been many steps in the right direction, such as the following:

(i) Foundation of the internationally active Virtual Muography Institute in Tokyo, Japan, on November 8, 2016.

(ii) The annual meetings of the General Assembly of Muographers.

(iii) Increasing numbers of muography-dedicated sessions in scientific conferences, symposiums, and workshops.

(iv) Increasing numbers of multidisciplinary and even transdisciplinary research (e.g., muography applied to challenges in geosciences, mining and civil engineering; geophysical joint inversions).

(v) Increasing numbers of publications combining the resources and expertise of previously separate research teams.

Yet, there are still many communities of muographers operating in isolation, although for some, this may be voluntary. Nevertheless, more collaborative actions and structural frameworks are needed to maintain the positive growth stage of muography and offer the newcomers alternatives for honing their new skills and nurturing their maturing interests to join this emerging discipline as active participants. Indeed, we claim that muography as a science cannot grow to its full potential unless the numbers of present-day muography developers are drastically increased by a much larger number of muography appliers.

Encouraging the potential muography appliers to make the leap for starting using muography for their application-specific challenges is a task that requires attention, at least if we seek to increase the appeal of muography. All steps listed above support this goal, but it remains to be seen whether these actions are enough to get muography widely recognized in the next five years or so. Hence, to support and speed up the maturing of muography, we propose that even more transdisciplinarity actions between present-day muographers (dominated by particle physicists and detector designers) and the various end-user disciplines are desirable. As an example, geoscience applications alone require collaboration with an extremely wide range of specialists, such as field geologists, structural geologists, petrologists, mineralogists, geochemists, ore geologists, mineral exploration experts, sedimentary geologists, geomorphologists, theoretical geophysicists, exploration geophysicists, seismologists, hydrologists, hydrogeophysicists, volcanologists, geohazard specialists, and numerous more. Likewise, also civil engineering applications require collaboration with a broad group of disciplines and stakeholders. Nevertheless, if the present-day muographers do their best and follow at least some of the suggested task points, muography has a bright future ahead. Towards the golden age of muography!

\section{CONFLICTS OF INTEREST}

The authors declare that there are no conflicts of interest regarding the publication of this paper.

\section{ACKNOWLEDGMENTS}

This work is APSI Contribution \#24. 


\section{References}

[1] G. H. Hadorn, The Emergence of Transdisciplinarity as a Form of Research. Handbook of Transdisciplinary Research, 19-39, Springer, Dordrecht (2008). doi:10.1007/978-1-4020-6699-3_2.

[2] M. Holma et al., Trends in publishing muography related research. The situation at the end of 2020, JAIS (2022). doi:10.xxxxxxxxxxxxxxx.

[3] J. Marteau et al., Development of Scintillator-Based Muon Detectors For Muography, Geophys. Monogr. Ser. 270 (2022, in print).

[4] K. Morishima et al., Development of Nuclear Emulsions for Muography, Geophys. Monogr. Ser. 270 (2022, in print).

[5] A. Bonneville et al., A novel muon detector for borehole density tomography. Nucl. Instrum. Meth. A 851, 108-117 (2017). doi:10.1016/j.nima.2017.01.023.

[6] S. Bouteille and S. Procureur, Development of Micro-Pattern Gaseous Detectors for Muography, Geophys. Monogr. Ser. 270 (2022, in print).

[7] S. Andringa et al., Resistive Plate Chambers in Muography, Geophys. Monogr. Ser. 270 (2022, in print).

[8] D. Varga et al., Development of Multi-Wire Proportional Chamber-Based Trackers for Muography, Geophys. Monogr. Ser. 270 (2022, in print).

[9] E. P. George, Cosmic rays measure overburden of tunnel, Commonwealth Engineer 43, 455-457 (1955).

[10] H. K. M. Tanaka et al., Detecting a mass change inside a volcano by cosmic-ray muon radiography (muography): First results from measurements at Asama volcano, Japan, Geophys. Res. Lett. 36, L17302 (2009). doi:10.1029/2009GL039448.

[11] H. K. M. Tanaka, Principles of Muography and Pioneering Works, Geophys. Monogr. Ser. 270 (2022, in print).

[12] M. Holma et al., Future Prospects of Muography for Geological Research and Geotechnical and Mining Engineering, Geophys. Monogr. Ser. 270 (2022, in print).

[13] Z.-X. Zhang et al., Muography and Its Potential Applications to Mining and Rock Engineering, Rock Mech Rock Eng 53, $4893-4907$ (2020). doi:10.1007/s00603-020-02199-9.

[14] E. Darian-Smith and P. McCarty, Beyond Interdisciplinarity: Developing a Global Transdisciplinary Framework, Transcience 7(2) (2016). 